\title{
Association between Non-Face-to-Face Interactions and Incident Disability in Older Adults
}

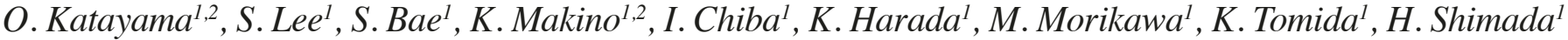

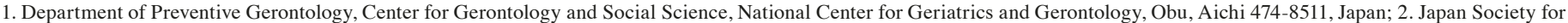
the Promotion of Science, Chiyoda-ku, Tokyo 102-0083, Japan.

Corresponding Author: Osamu Katayama, National Center for Geriatrics and Gerontology, Obu City, Aichi Japan, katayama.o@ncgg.go.jp

\begin{abstract}
OBJECTIVES: This observational prospective cohort study, conducted between September 2015 and February 2019, aimed to investigate the association between the incidence of disability and non-face-to-face interactions among community-dwelling older adults in Japan.

DESIGN: Participants reported their interaction status using a selfreport questionnaire. Face-to-face interactions comprised in-person meetings, while virtual interactions (e.g., via phone calls or emails) were defined as non-face-to-face interactions. We examined the relationship between their interaction status at baseline and the risk of disability incidence at follow-up. We also considered several potential confounding variables, such as demographic characteristics.

SETTING: The National Center for Geriatrics and Gerontology-Study of Geriatric Syndromes.

PARTICIPANTS: We included 1159 adults from Takahama City aged $\geq 75$ years (mean age \pm standard deviation $=79.5 \pm 3.6$ years).

MEASUREMENTS: Interaction status was assessed using a selfreported questionnaire consisting of two sections (face-to-face and non-face-to-face interactions), and four questionnaire items. Based on the responses we categorized study participants into four groups: "both interactions," "face-to-face only," "non-face-to-face only," and "no interactions."

RESULTS: Individuals with both kinds of interactions $(49.3 / 1000$ person-years) or only one kind of interaction (face-to-face $=57.7 / 1000$ person-years; non-face-to-face $=41.2$ person-years) had lower incidence of disability than those with no interactions $(88.9 / 1000$ person-years). Moreover, the hazard ratios adjusted for potential confounding factors for the incidence of disability in the both interaction, face-to-face-only, and non-face-to-face only groups were 0.57 (confidence interval $=0.39-0.82 ; \mathrm{p}=0.003), 0.66$ (confidence interval $=0.44-0.98 ; \mathrm{p}=0.038$ ), and 0.47 (confidence interval $=0.22$ $0.99 ; \mathrm{p}=0.048)$, respectively.

CONCLUSION: Considering the interaction status of older adults in their day-to-day practice, clinicians may be able to achieve better outcomes in the primary prevention of disease by encouraging older adults to engage in any form of interaction, including non-face-to-face interactions.
\end{abstract}

Key words: Communication, community dwelling, disability studies, older adults, social interaction.

\section{Introduction}

Tapan is expected to have the largest proportion of older adults worldwide by 2050 with $39.9 \%$ of the national population projected to be aged $>65$ years (1). The population of the older population ( $\geq 65$ years) reached 35.25 million individuals in March 2019, with 17.3 million adults aged $<75$ years and 17.96 million adults aged $\geq 75$ years (2). In developed nations faced with an aging population (including Japan), many of these older adults require care $(1,3)$. Since the introduction of Japan's long-term care insurance (LTCI) system in 2000, the number of older adults requiring this service has increased. The Japanese LTCI system has been operating for approximately 20 years and currently serves nearly 6.58 million people (4). In terms of percentage of senior adults aged $\geq 65$ who have been certified as requiring long-term care, 0.73 million (women; 0.37 million) are aged $<75$ years and 5.72 million (women; 4.08 million) are aged $\geq 75$ years (2). Therefore, the number of people certified as needing long-term care increases significantly after 75 years of age (5). Dementia, cerebrovascular disease, and age-related weaknesses account for about half the factors that cause disability in the older adults of both sexes in Japan (5). Community-dwelling older adults in Japan with physical frailty, a typical example of age-related weakness, were at higher risk of requiring care in the LTCI system, and the total cost of LTCI incurred was correspondingly higher for these adults (6). The rapid aging of populations in some countries has led to a growing increase in the number of older disabled adults. This exacerbates the need for long-term care and meeting the associated costs, which is typically borne by the families of the disabled older adults and governments (7). Therefore, it is necessary to clarify the risk of disability in people aged $\geq 75$ years.

Studies are increasingly focusing on the impact of social interaction on the health of older adults. Social activity can include interactions with the environment and ingroup members (e.g. like-minded people) and can engage the mind and body (8). Previous studies showed that participation in social activities is associated with a reduced risk of developing disability in the future $(9,10)$. However, due to the recent global spread of coronavirus disease (COVID-19), opportunities for face-to-face interactions with other people are decreasing worldwide (11). A study conducted before and 
during the first wave of COVID-19 outbreaks in Japan (January to April 2020) showed that the duration of physical activity in older adults decreased by approximately $30 \%$ after the first wave of COVID-19 in Japan (12). Similarly, the number of steps taken by older Japanese adults decreased by up to $30 \%$ after the initial spread of COVID-19 (13). This has fueled concerns that the incidence of disability may increase after the COVID-19 pandemic due to a decrease in daily physical activity (12). Interestingly, a previous study indicated that the daily physical activity time recovered after the spread of COVID-19 (at June 2020) up to pre-infection levels (at January 2020), although recovery was difficult when living alone and being socially inactive (14).

Even before the COVID-19 pandemic, studies reported that one in four older adults was socially isolated and more than $40 \%$ experienced loneliness (15). Decades of observational studies have demonstrated the long-term negative health outcomes of social isolation and loneliness $(16,17)$. This highlights the importance of non-face-to-face interactions such as communication via telephone and video calls (15). According to a Japanese government report on the use of social networking services (SNS) by older Japanese adults in 2019, $51.7 \%$ of those aged 60-69 years, $40.7 \%$ of those aged $70-79$ years, and $42.8 \%$ of those aged 80 years and above used SNS, and $86.9 \%$ of their use time was to communicate with acquaintances (18).

Therefore, non-face-to-face interactions with friends and acquaintances may prevent disability among older adults when face-to-face interaction is not possible because of the spread of COVID-19. However, there is insufficient evidence to determine whether non-face-to-face interactions are associated with a reduced risk of disability in the older Japanese population. Therefore, this study aimed to clarify whether nonface-to-face interactions are associated with a reduced risk of disability in community-dwelling adults aged $\geq 75$ years who are at high risk of disability incidence. We hypothesized that the risk of disability incidence would be lower among older adults who engage in non-face-to-face interaction, as opposed to those who do not engage in either face-to-face or non-face-to-face interactions.

\section{Methods}

\section{Study design}

This was an observational prospective cohort study of adults enrolled in a population-based cohort study, which is part of the National Center for Geriatrics and Gerontology-Study of Geriatric Syndromes (NCGG-SGS). The NCGG-SGS is a cohort study with the primary goal of establishing a screening system for geriatric syndromes and validating evidence-based interventions for preventing geriatric syndromes (19). This study investigated the association between non-face-to-face interaction at the baseline and the incidence of disability during a mean follow-up of 33.7 months (standard deviation [SD] = 8.3 months) from baseline.

\section{Participants}

Overall, 1352 community-dwelling older adults participated in the assessments, which included face-to-face interviews and physical and cognitive function evaluations. We included participants who resided in Takahama City who were aged $\geq 75$ years at the time of the study (September 2015 to February 2017). We excluded data from the following sets of participants: (1) individuals who had health problems that critically affected disability incidence (such as dementia, Parkinson's disease, and a history of strokes $)(n=116)$, based on face-to-face interviews conducted by a qualified nurse; (2) individuals who needed support or care, as certified by the Japanese public LTCI system, due to disability $(\mathrm{n}=67)$; (3) individuals with disabilities affecting basic activities of daily living (ADLs) $(\mathrm{n}=$ 3 ); and (4) individual with responses with missing variables of exclusion criteria $(n=7)$. Of the initial 1352 participants, 193 were excluded based on these criteria. The study participants were followed up from September 2015 to February 2019, with a mean follow-up of 33.7 months ( $\mathrm{SD}=8.3$ months). All participants provided written informed consent prior to participating in the study. This study was approved by the Ethics Committee of the National Center for Geriatrics and Gerontology (Approval Number: 1440-3). Using an opt-out approach, we disclosed information about this study and excluded data when the participants declined to participate directly or via proxy.

\section{Measurement of face-to-face and non-face-to-face interaction}

Interaction status was assessed using a self-reported questionnaire consisting of two sections (face-to-face and non-face-to-face interactions), and four questionnaire items. Questionnaire items were adapted from the NCGG-SGS (20, 21). The face-to-face interaction items included: (1) "Do you eat out or participate in a tea party with friends?" and (2) "Do you go shopping with friends?" Interaction frequency was assessed by participants as "never," "once a month or less," "several times a month," "1-2 times per week," "3-6 times per week," and "every day." Individuals who answered "never" to both questions were classified as having no face-to-face interaction, while those who picked any other option for either question were classified as having face-to-face interaction. The non-face-to-face interaction items included: (3) "How many days in a week do you call or email your friends?" and (4) "How many days in a week do you call or email acquaintances (neighbors, business associates, etc.)?" Interaction frequency was assessed by interaction as "0-1 day," "2-3 days," "4-5 days," and "6-7 days." Individuals who answered "0-1 day" to both questions were classified as having no non-face-to-face interaction, while those who answered other than " $0-1$ day" to either question were classified as having non-face-to-face interaction. Then, based on these responses, we categorized study participants into four groups: the group that conducted both face-to-face and non-face-to-face exchanges was labeled as having "both interactions," the group that conducted only 
face-to-face interactions was labeled "face-to-face only," the only non-face-to-face interactions group was labeled "non-faceto-face only," and the group that did not conduct face-to-face or non-face-to-face interactions was labeled as having "no interactions."

\section{Disability determination}

Participants were tracked monthly for a new incidence of LTCI certification, as recorded by the Japanese LTCI system and measured by each municipal government. The LTCI system classifies a person in "Support Level 1 or 2 " to indicate a need for assistance to support ADLs, or in "Care Levels 1 through 5 " to indicate a need for continuous care (22). In this study, disability was defined as any LTCI certification level, and we defined disability onset as the point at which a participant received LTCI certification.

\section{Potential confounding factors}

Potential confounding variables included demographic variables, chronic disease, cohabitation status, and paid work status that may be independently associated with disability in older adults $(9,10,21,23)$. Therefore, our model included the following covariates: age at enrollment, sex, presence of chronic diseases, medication use, body mass index (BMI), cohabitation status, and work. Self-reported presence of chronic diseases (heart disease, diabetes, and hypertension) through face-to-face interviews with nurses were also included as covariates. Cohabitation status assessment was conducted by staff, who were trained by the study authors. The participants were asked to self-report their work status using a questionnaire.

\section{Statistical analysis}

One-way analysis of variance and Pearson's chi-square tests were used to compare variables among groups of participants who were disability-free, who had a disability, and those who died or relocated. Similarly, the aforementioned tests were used to compare predictor variables among participants who engaged in "both interactions," "face-to-face only" interaction, "non-face-to-face only" interaction, and "no interaction" (group labels in quotation marks). Adjusted standardized residuals $>1.96$ indicated $\mathrm{P}<0.05$. Sensitivity analyses were performed to evaluate whether a potential bias could be introduced by the censoring mechanism for persons who died or relocated. We calculated the cumulative incident disability during follow-ups for each of the four above-mentioned interaction groups using Kaplan-Meier curves. Intergroup differences were estimated using the log-rank test. Crude and adjusted Cox proportional hazard models were constructed to calculate hazard ratios (HRs) with 95\% confidence intervals (CIs) for incident disability risk. The adjusted model was adjusted for covariates known to be associated with disability in previous studies; age, sex, heart disease, diabetes, hypertension, medication use, BMI, cohabitation status, and work $(9,10,21,23)$. The significance level was set at $\mathrm{P}<0.05$. All analyses were performed using
IBM SPSS (version 25.0; IBM Japan, Tokyo).

\section{Results}

The final analysis included data from 1159 older adults (666 women; mean age \pm SD $79.5 \pm 3.6$ years; age range $=$ 75-96 years). Among 1159 participants, 917, 188, and 54 participants remained disability-free, developed a disability, and either died or relocated, respectively. Table 1 shows the baseline characteristics of the study participants who remained disability-free, developed a disability, and died or relocated. At the baseline, participants who had died or relocated were older than those who were successfully followed up with and remained disability-free $(\mathrm{P}<0.001)$; participants who developed a disability were older and had not worked than those who were followed up with and remained disability-free $(\mathrm{P}<0.05)$. Table 2 shows the baseline characteristics of the study participants according to interaction status. Significant differences between the four interaction groups were found for participant age, sex, and paid work status $(\mathrm{P}<0.05)$.

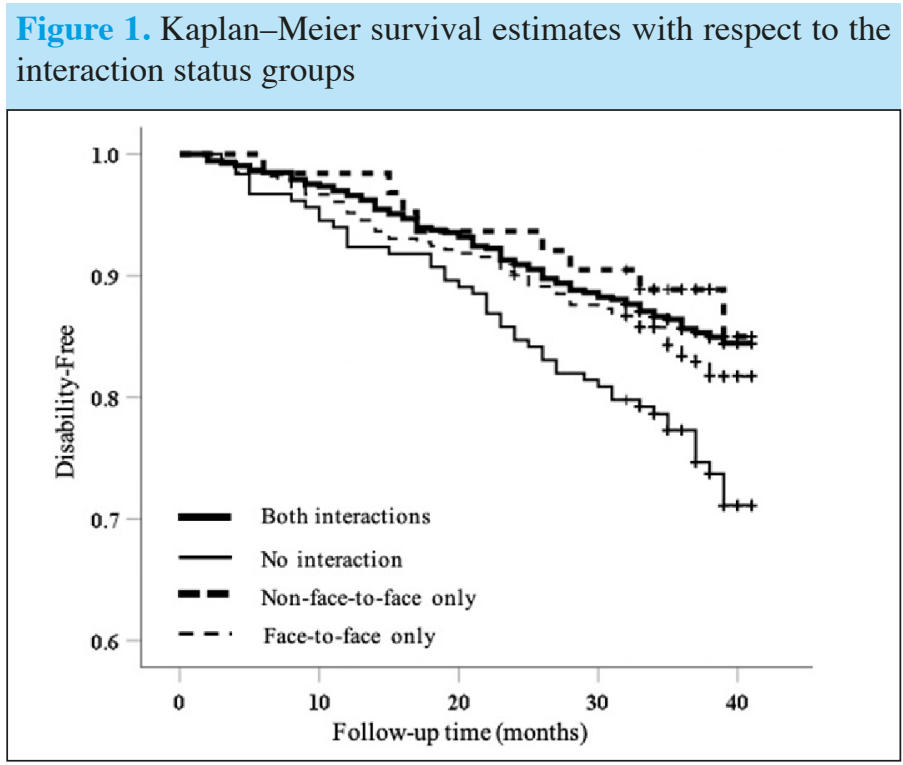

Note: The participants who belonged to the no interaction group had a lower probability of being disability-free than those who belonged to the other groups.

The incidence of disability was 49.3 per 1000 personyears for those who in the "both interactions" group, 57.7 per 1000 person-years for those in the "face-to-face only" group, and 41.2 per 1000 person-years for those in the "not faceto-face only" group, compared with $88.9 / 1000$ person-years for participants in the "no interaction" group. In Figure 1, the Kaplan-Meier survival estimates show that participants who were involved in both face-to-face and non-face-to-face interactions had a higher probability of being disability-free than those who did not $(\mathrm{P}=0.001, \mathrm{P}=0.021$, and $\mathrm{P}=0.036$, respectively). The potential confounder-adjusted disability HRs for participants in the "both interactions," "face-to-face only," and "non-face-to-face only" groups were 0.57 (CI, 0.39-0.82; $\mathrm{P}=0.003), 0.66$ (CI, 0.44-0.98; $\mathrm{P}=0.038)$, and 0.47 (CI, 0.22$0.99 ; \mathrm{P}=0.048$ ), respectively (Table 3 ). 
Table 1. Baseline characteristics of study participants by follow-up status

\begin{tabular}{|c|c|c|c|c|c|c|}
\hline Variable & $\begin{array}{c}\text { Total } \\
(\mathbf{n}=\mathbf{1 1 5 9})\end{array}$ & $\begin{array}{l}\text { Participants Free } \\
\text { of Disability } \\
(\mathbf{n}=917)\end{array}$ & $\begin{array}{l}\text { Participants } \\
\text { with Disability } \\
\quad(\mathbf{n}=188)\end{array}$ & $\begin{array}{c}\text { Participants Who } \\
\text { Died or Relocated } \\
\quad(n=54)\end{array}$ & p-Value & Post Hoc \\
\hline Mean age at baseline, $y$ & $79.5 \pm 3.6$ & $79.0 \pm 3.2$ & $81.8 \pm 3.9$ & $81.5 \pm 4.7$ & $<0.001 *$ & $\begin{array}{c}\text { Free }<\text { Disability, Died } \\
\text { or relocated }\end{array}$ \\
\hline Sex, Female (\%) & $666(57.5)$ & $527(57.5)$ & $112(59.6)$ & $27(50.0)$ & $0.455 \dagger$ & \\
\hline Medication use, $\mathrm{n}$ & $3.6 \pm 2.7$ & $3.6 \pm 2.7$ & $3.7 \pm 2.6$ & $4.2 \pm 3.0$ & $0.228 *$ & \\
\hline Diabetes, no (\%) & $991(85.5)$ & 785 (85.6) & $163(86.7)$ & $43(85.5)$ & $0.421 \dagger$ & \\
\hline Hypertension, no (\%) & 485 (41.9) & $383(41.8)$ & $81(43.3)$ & $21(38.9)$ & $0.835 \dagger$ & \\
\hline $\mathrm{BMI}, \mathrm{kg} / \mathrm{m} 2$ & $23.4 \pm 3.1$ & $23.1 \pm 3.7$ & $23.3 \pm 4.2$ & $23.3 \pm 3.2$ & $0.517 *$ & \\
\hline Cohabitation status, yes (\%) & $165(14.2)$ & $125(13.6)$ & $34(18.1)$ & $6(11.1)$ & $0.225 \dagger$ & \\
\hline Work, yes $(\%)$ & $206(17.8)$ & $176(19.2) \ddagger$ & $21(11.2) \S$ & $9(16.7)$ & $0.031 \dagger$ & \\
\hline Non-face-to-face interaction, $\mathrm{n}(\%)$ & $68(5.9)$ & $55(6.0)$ & $8(4.3)$ & $5(9.3)$ & & \\
\hline No interaction, $\mathrm{n}(\%)$ & $194(16.7)$ & $136(14.8) \S$ & $47(25.0) \ddagger$ & $11(20.4)$ & & \\
\hline
\end{tabular}

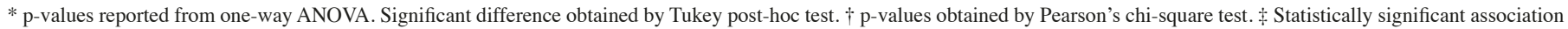

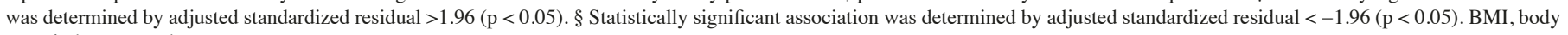
mass index; $n$, number; $y$, years.

Table 2. Baseline characteristics of the study participants by interaction status

\begin{tabular}{|c|c|c|c|c|c|c|}
\hline Variable & $\begin{array}{l}\text { Both interactions } \\
\quad(\mathbf{n}=\mathbf{5 5 0})\end{array}$ & $\begin{array}{l}\text { Face-to-face interaction } \\
\qquad(\mathrm{n}=\mathbf{3 4 7})\end{array}$ & $\begin{array}{c}\text { Non-face-to-face } \\
\text { interaction } \\
(n=68)\end{array}$ & $\begin{array}{l}\text { No interactions } \\
\quad(n=194)\end{array}$ & p-Value & Post Hoc \\
\hline Mean age at baseline, $y$ & $79.3 \pm 3.4$ & $79.6 \pm 3.7$ & $79.4 \pm 3.3$ & $80.2 \pm 4.1$ & $0.030 *$ & Both $<$ No \\
\hline Medication use, $\mathrm{n}$ Chronic disease & $3.7 \pm 2.7$ & $3.6 \pm 2.6$ & $3.9 \pm 2.7$ & $3.7 \pm 2.9$ & $0.844 *$ & \\
\hline Heart disease, no (\%) & $432(78.5)$ & $282(81.3)$ & $53(77.9)$ & $159(82.0)$ & $0.635 \dagger$ & \\
\hline Hypertension, no (\%) & $218(39.7)$ & $150(43.2)$ & $27(39.7)$ & $90(46.4)$ & $0.380 \dagger$ & \\
\hline $\mathrm{BMI}, \mathrm{kg} / \mathrm{m} 2$ & $23.6 \pm 3.3$ & $23.1 \pm 3.1$ & $23.3 \pm 3.4$ & $22.7 \pm 3.1$ & $0.003 *$ & No $<$ Both \\
\hline Cohabitation status, yes (\%) & $460(83.6)$ & $308(88.8)$ & $58(85.3)$ & $168(86.6)$ & $0.194 \dagger$ & \\
\hline Work, yes (\%) & $116(21.1) \ddagger$ & $43(12.4) \S$ & $16(23.5)$ & $31(16.0)$ & $0.005 \dagger$ & \\
\hline
\end{tabular}

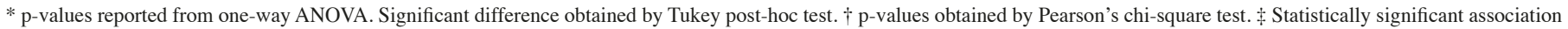

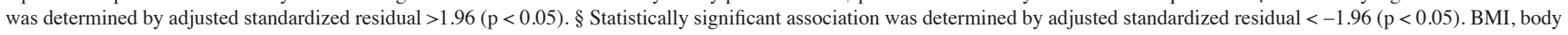
mass index; CI, confidence interval; n, number; y, years.

\section{Discussion}

In this observational, prospective population-based cohort study, we found that those involved in both face-to-face and non-face-to-face interactions (49.3/1000 person-years), those with only face-to-face interactions (57.7/1000 person-years), and only non-face-to-face interactions (41.2/1000 personyears) had lower incidence rates of disability compared to those with no interaction (88.9/1000 person-years). The potential confounding factors adjusted HRs for incidence of disability in the both interactions, face-to-face-only, and non-faceto-face only groups were 0.57 (CI, 0.39-0.82; $\mathrm{P}=0.003$ ), 0.66 (CI, 0.44-0.98; $\mathrm{P}=0.038$ ), and 0.47 (CI, 0.22-0.99; P
$=0.048)$, respectively; these correspond to a $42 \%, 34 \%$, and $53 \%$ decrease in the disability risk of participants with both interactions, face-to-face only interactions, and non-face-to-face only interactions, respectively.

Several longitudinal studies have shown that a greater level of participation in social activities is related to a lower risk of incident functional disability $(24,25)$. Persons who participate in social activities have an opportunity to communicate and collaborate with others (26). Therefore, the risk of developing disability may be reduced in the groups that were involved in face-to-face interactions. These results support the findings of previous studies that social activities reduce the risk of incident functional disability $(24,25)$. Our study uncovered an important association between the incidence of disability and non-face-to- 
Table 3. Cox proportional hazard models of the relationships between interaction status and incident disability

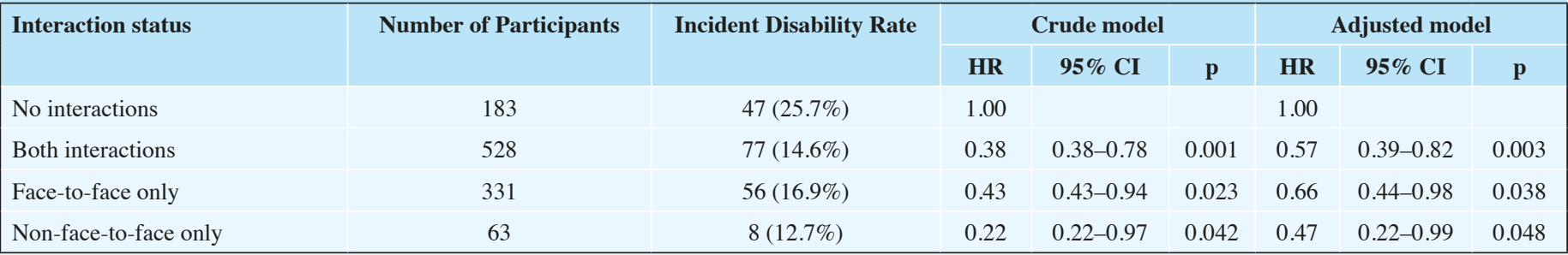

Adjusted model is adjusted for age, sex, medication, heart disease, diabetes, hypertension, BMI, cohabitation status, and work.

face interaction in older adults aged $>75$ years; this is a novel finding.

The most frequently used SNS in older adults is LINE (a popular message application in Japan). A previous study demonstrated that frequent usage of LINE (both posting and checking) among older adults was independently associated with better well-being (27). We also found that high activity on Facebook Messenger may also have a positive impact on depressed mood (28). Older adults aged $>75$ years who engage in non-face-to-face interactions may have the same opportunities to communicate, cooperate, and support others as they do when participating in face-to-face social activities. However, we did not have access to the applications and content used for non-face-to-face interaction; this is a promising area for future investigation.

Our findings suggest that even non-face-to-face interactions may reduce the risk of social inactivity-related disability in the same way as face-to-face interactions. To the best of our knowledge, this is the first study to evaluate and contrast the impact of face-to-face and non-face-to-face interactions on the relationship between interaction status and disability incidence. Since the start of the COVID-19 pandemic in February 2020, opportunities for face-to-face interactions have decreased in Japan, and more broadly, worldwide (11). The COVID-19 crisis has exacerbated the challenges, with increasing social isolation and loneliness among those who live alone and may even cause a decline in the well-being of older adults with previously active or healthy social lives (15). The results of this study support the original hypothesis that older adults who engage in non-face-to-face interaction alone have a lower risk of disability incidence than older adults who engage in neither face-to-face nor non-face-to-face interaction (in other words, who do not participate in any form of interaction). Our findings suggest that non-face-to-face interaction may reduce the risk of disability among Japanese adults aged $\geq 75$ years who are at high risk of developing disability during a period when face-to-face interaction is reduced due to the spread of COVID-19. However, in this study, we were not able to perform a detailed assessment and analysis to adjust for important confounding factors influencing the development of disability, such as exercise, daily activity patterns, nutritional status, and daily intake. This aspect should be verified in the future. Among the participants in this study, it was more common for men to engage in non-face-to-face interactions. Since the life expectancy of Japanese women is higher than that of men, women are more likely to live alone in Japan. In addition to the aftermath of COVID-19, this may suggest that establishing safe face-to-face interactions and enforcing measures that allow women to have more opportunities for non-face-to-face interactions may be necessary for disability prevention. Additionally, group rehabilitation exercises linked to the therapist in one site, with group members in their own homes may be arranged. Likewise, other group therapies may also be conducted.

A central strength of this study is the large sample size of study participants aged $>75$ years. In addition to this, the operationalized assessment to identify non-face-to-face interactions and risk of disability provided a high level of standardization across study participants. Finally, by classifying the interaction status into four groups, we were able to compare and contrast the impact of face-to-face interactions with nonface-to-face interactions on disability incidence. However, this study also had some limitations. First, we sent participation invitation letters to individuals who lived in Takahama City, who were not hospitalized or in residential care, who were not certified by the LTCI system as having a functional disability, or who were not participating in another study. The participants in our study were older adults who had access to health checkups from their homes; this implies that the sample excluded people with other conditions. Second, we tracked the monthly incidence of new LTCI certifications as recorded by the Japanese LTCI system which was measured by the municipality. However, information on the causes of disability at follow-up was not available. Additionally, medications used vary with time, and participants may have been on different medications at follow-up than at baseline. Moreover, the participant may have been hospitalized for acute illnesses that create a strong risk for disability. Thus, we did not know whether the older adults during the follow-up period had developed additional diseases; therefore, future studies should account for such variables. Third, sensory function is also important in the process of physical and mental decline; however, we did not conduct a detailed assessment of sensory function. In future studies, we intend to perform a detailed evaluation and adjust it using a statistical model. Finally, we did not have access to the applications and content used for nonface-to-face interactions, and these aspects are being assessed in a longitudinal study.

\section{Conclusion}

Although our study leaves room for further investigation, we found that there may be an important association between 
the incidence of disability and non-face-to-face interactions in older adults aged $>75$ years. These findings suggest that even non-face-to-face interactions may reduce the risk of disability from social activities the way face-to-face interactions do. Given the increasingly high prevalence of disability and its strong association with numerous adverse health outcomes, clinicians may be able to achieve better outcomes in the primary prevention of disease by encouraging older adults to engage in non-face-to-face interactions by including an older adult's interaction status in their day-to-day medical practice.

Acknowledgments: We thank the healthcare staff for their assistance with the assessments. We would like to thank Editage for English language editing. This work was funded by Research Funding for Longevity Sciences (27-22) from the National Center for Geriatrics and Gerontology (NCGG), a Grant-in-Aid for Scientific Research (A) (26242059), and a Grant-in-Aid for JSPS Fellows (21 J01605). In addition, R\&D expenses were commissioned by Takahama City. The funders did not have a role in the study design, in the collection, analysis, and interpretation of data, in the writing of the report, or in the decision to submit the article for publication.

Ethical Standards: The study protocol was approved by the Ethics Committee of the National Center for Geriatrics and Gerontology (No. 1440-3).

Disclosure Statement: The authors declare no conflict of interest.

Data Availability Statement: Data available on request from the authors.

\section{References}

1. United Nations Department of Economic and Social Affairs Population Division World population prospects: the 2019 revision population database [http://esa.un.org/ unpd/wpp/index.htm]. 2020 (accessed on 1 May 2020).

2. Ministry of Health, Labour and Welfare. Kaigohoken jigyou jyokyo houkoku. 2020 (accessed on 1 June 2021)

3. Christensen K, Doblhammer G, Rau R, Vaupel JW. Ageing populations: the challenges ahead. Lancet. 2009;374:1196-1208.

4. Ministry of Health, Labour and Welfare. Report on various current states around LTCI (Kaigo hoken seido wo meguru genjonitsuite). 2019 (accessed on 1 May 2020).

5. Japan CO. Annual Report on the Ageing Society. 2020.

6. Makizako H, Shimada H, Tsutsumimoto K, et al. Physical Frailty and Future Costs of Long-Term Care in Older Adults: Results from the NCGG-SGS. Gerontology. 2021:110 .

7. Zeng Q, Wang Q, Zhang L, Xu X. Comparison of the Measurement of Long-Term Care Costs between China and Other Countries: A Systematic Review of the Last Decade. Healthcare (Basel). 2020;8.

8. Arai S, Pedlar A. Moving beyond individualism in leisure theory: a critical analysis of concepts of community and social engagement. Leisure Studies. 2003;22:185-202.

9. Hikichi H, Kondo N, Kondo K, Aida J, Takeda T, Kawachi I. Effect of a community intervention programme promoting social interactions on functional disability prevention for older adults: propensity score matching and instrumental variable analyses, JAGES Taketoyo study. J Epidemiol Community Health. 2015;69:905-910.
10. Makizako H, Shimada H, Tsutsumimoto K, et al. Social Frailty in CommunityDwelling Older Adults as a Risk Factor for Disability. J Am Med Dir Assoc. 2015;16:1003 e1007-1011.

11. Ministry of Health, Labour and Welfare. Roujin hoken kenko zoushintou jigyou. 2020(accessed on 3 June 2021).

12. Yamada M, Kimura Y, Ishiyama D, et al. Effect of the COVID-19 Epidemic on Physical Activity in Community-Dwelling Older Adults in Japan: A Cross-Sectional Online Survey. J Nutr Health Aging. 2020;24:948-950.

13. Tison GH, Avram R, Kuhar P, et al. Worldwide Effect of COVID-19 on Physical Activity: A Descriptive Study. Ann Intern Med. 2020.

14. Yamada M, Kimura Y, Ishiyama D, et al. Letter to the Editor: Recovery of Physical Activity among Older Japanese Adults since the First Wave of the COVID-19 Pandemic. J Nutr Health Aging. 2020;24:1036-1037.

15. Cudjoe T, Kotwal A. "Social distancing" amidst a crisis in social isolation and loneliness. J Am Geriatr Soc. 2020.

16. National Academies of Sciences E, Medicine. Social isolation and loneliness in older adults: Opportunities for the health care system. National Academies Press; 2020.

17. Cudjoe T, Roth D, Szanton S, Wolff J, Boyd C, Thorpe Jr R. The epidemiology of social isolation: National health and aging trends study. The Journals of Gerontology: Series B. 2020;75:107-113.

18. Ministry of Internal Affairs and Communications J. Results of FY2019 Communication Usage Trend Survey. 2020 (accessed on 4 May 2020).

19. Shimada H, Makizako H, Lee S, et al. Impact of Cognitive Frailty on Daily Activities in Older Persons. J Nutr Health Aging. 2016;20:729-735.

20. Bae S, Lee S, Harada K, et al. Engagement in Lifestyle Activities is Associated with Increased Alzheimer's Disease-Associated Cortical Thickness and Cognitive Performance in Older Adults. J Clin Med. 2020;9.

21. Katayama O, Lee S, Bae S, et al. Participation in Social Activities and Relationship between Walking Habits and Disability Incidence. J Clin Med. 2021;10.

22. Tsutsui T, Muramatsu N. Care-needs certification in the long-term care insurance system of Japan. J Am Geriatr Soc. 2005;53:522-527.

23. Shimada H, Makizako H, Doi T, Tsutsumimoto K, Suzuki T. Incidence of Disability in Frail Older Persons With or Without Slow Walking Speed. J Am Med Dir Assoc. 2015;16:690-696.

24. James B, Boyle P, Buchman A, Bennett D. Relation of late-life social activity with incident disability among community-dwelling older adults. J Gerontol A Biol Sci Med Sci. 2011;66:467-473

25. Kanamori S, Kai Y, Aida J, et al. Social participation and the prevention of functional disability in older Japanese: the JAGES cohort study. PLoS One. 2014;9:e99638.

26. Levasseur M, Richard L, Gauvin L, Raymond E. Inventory and analysis of definitions of social participation found in the aging literature: proposed taxonomy of social activities. Soc Sci Med. 2010;71:2141-2149.

27. Sakurai R, Nemoto Y, Mastunaga H, Fujiwara Y. Who is mentally healthy? Mental health profiles of Japanese social networking service users with a focus on LINE, Facebook, Twitter, and Instagram. PLoS One. 2021;16:e246090.

28. Frison E, Eggermont S. Exploring the relationships between different types of Facebook use, perceived online social support, and adolescents' depressed mood. Social Science Computer Review. 2016;34:153-171.

How to cite this article: O. Katayama, S. Lee, S. Bae, et al. Association between NonFace-to-Face Interactions and Incident Disability in Older Adults. J Nutr Health Aging. 2022;26(2):147-152, http://dx.doi.org/10.1007/s12603-022-1728-5 